\title{
Enhanced Quality of Service in Worldwide Interoperability for Microwave Access Networks
}

\author{
Gihad yousif gafar mohammed ${ }^{1}$, Amin babiker abd.elnabi Mustafa ${ }^{2}$ \\ ${ }^{12}$,Department of Communications, Faculty of Engineering
}

\begin{abstract}
The WiMAX system effectively supports wide variety of broadband wireless access technologies, which including high speed internet and multimedia access with high Quality of service (QoS) requirements. Real time services such as VoIP are becoming popular and are major revenue earners for network service providers. However, there are still many challenges that need to be addressed to provide a steady and good quality voice connection over the best effort WiMAX network. To support flexibility, efficiency and various requirements of QoS over a range of different applications and environments several provisioning and mechanisms are provided. This research paper investigates the performance of VoIP traffic over WiMAX networks, the impact of various voice codec schemes and statistical distribution for VoIP over WiMAX has been investigated in detail. Through various simulation experiments under realistic networking scenarios, this study provides an insight into the VoIP performance in the WIMAX networks. The simulations results indicate that better choice of voice codec's and statistical distribution have significant impact on VoIP performance in the WiMAX networks and Performance of selected parameters will be done using the network simulator OPNET Modeler.
\end{abstract}

\section{Objective}

The objective of study is to guarantee QoS for multiple service class traffic in a multiple connection environment and to examine a case of QoS deployment over a cellular WiMAX network. In particular, the paper compares the performance obtained using two different QoS configurations differing from the delivery service class ,to guarantee QoS for multiple service class traffic in a multiple connection environment in WiMAX network the Adaptive modulation and coding scheme is used at the physical layer that adapts to the scheduled traffic to stabilize the QoS requirements of different traffic classes, Configure QoS mechanisms to guarantee low delay for multimedia application without drastically affecting data traffic. We analyze the distribution impact on traffic arrival time to quality of VoIP in WiMAX network; analysis the quality of service (QoS) with VoIP over WiMAX will be performed. Performance of selected parameters will be done using the network simulator, OPNET Modeler

\section{Problem statement}

The demand for multimedia applications in WiMAX networks is growing at a rapid pace. A method for guaranteeing Quality of Service (QoS) for different classes of traffic is therefore gaining importance. Hence designing and analyzing multimedia traffic and QoS parameters has become central to this problem. In this study, we ]have investigated the data and voice support in the WiMAX network and to examine the capability of a WiMAX network to deliver adequate QoS to voice and data applications.

\section{Research Methodology}

In our case, we have used OPNET Modeler and the study in presents a simulation model to analyzes the performance of an IEEE 802.16 system by focusing on the MAC layer scheduling and evaluate VoIP traffic by using G.729, G .711 and G .723 codecs.

\section{Literature Review} over WiMAX.

There has been quite a lot of research done in the past, involving models to Improve QoS Performance

Supporting real-time applications over any wireless network for example, $3 \mathrm{G}$ cellular networks, IEEE 802.11-based wireless LANs, and IEEE 802.16-based WIMax) poses many challenges, including limited bandwidth, coping with bandwidth fluctuations, and lost or corrupted data. Due to the growing popularity of streaming services over wireless networks, the problems have been well researched and many solutions have been proposed which combine audio and video processing techniques with mechanisms that are usually dealt with in the data link and physical layers. These approaches can broadly be classified into two categories: automatic repeat request (ARQ) and forward error correction (FEC). ARQ schemes provide high reliability when the channel is good or moderate. However, for error-prone channels, the throughput drops due to the 
increased frequency of retransmissions. In order to counter this effect, hybrid ARQ schemes are used which combine the FEC with the ARQ schemes.

As far as VoIP is concerned, an assessment of the Internet in supporting toll-quality telephone calls was conducted in [1]. In order to provide good service and to meet the user demands, research has been in progress both in wireless technologies and VoIP network system. VoIP is becoming more and more popular especially after the deployment of WiMAX network in many countries [2]. Different aspects of VoIP over WiMAX have been addressed by researchers.

The authors in [3] have investigated the data and voice support in the WiMAX network. The aim of their work was to examine the QoS deployment over WiMAX network and compare the performance obtained using two different WiMAX services classes i.e. UGS and ertPS. Supporting real-time applications over any wireless network (for example, 3G cellular networks, IEEE 802.11-based wireless LANs, and IEEE 802.16based WiMax) poses many challenges, including limited bandwidth, coping with bandwidth fluctuations, and lost or corrupted data. Due to the growing popularity of streaming services over wireless networks, the problems have been well researched and many solutions have been proposed which combine audio and video processing techniques with mechanisms that are usually dealt with in the data link and physical layers. These approaches can broadly be classified into two categories: automatic repeat request (ARQ) and forward error correction (FEC). ARQ schemes provide high reliability when the channel is good or moderate. However, for error-prone channels, the throughput drops due to the increased frequency of retransmissions. In order to counter this effect, hybrid ARQ schemes are used which combine the FEC with the ARQ schemes.

As far as VoIP is concerned, an assessment of the Internet in supporting toll-quality telephone calls was conducted in [4]. in [5] studied performance of scheduling algorithms for WiMAX networks. Some of their work are quite related with our works. However they focused mainly on implementing some existing scheduling algorithms.

The purpose of this study was to examine a case of QoS deployment over a cellular WiMAX network and to examine the capability of a WiMAX network to deliver adequate QoS to voice and data applications. The methodologies taken include creating the WiMAX network, deploying the required applications, deploying QoS configurations within the WiMAX last-mile, adjusting the QoS configurations within the WiMAX cells to meet voice requirements, and further adjusting the QoS configurations to improve data application performance, without degrading the performance of voice. This topic was identified as being importance to researcher and manufacturers in providing them the necessary background for theirworks. The paper is organized as follows. Section 2 explains the QoS in IEEE 802.16. Section 3 provides an overview of VoIP including R-Score and MOS. Section 4 provides the design of system model. The results are presented in Section 5. Finally, Section 6 concludes the paper and according to this study provide d good result. In [6], a study was conducted, where active and passive traffic measurements were taken to identify the issues involved with the deployment of voice services over the IP network. The results show that QoS differentiation is not needed in the current backbone, but new protocols and mechanisms need to be introduced to provide better protection against link failures. The reason is that link failures are followed by long periods of routing instability during which packets are dropped because of being forwarded along invalid paths. The study in [7] presents a simulation model and analyzes the performance of an IEEE 802.16 system by focusing on the MAC layer scheduling for VoIP traffic using AMR codecs. However, for IP networks, the aggregate background traffic affects the performance of VoIP. A rapid growth has been noticed in various wireless technologies in recent years.

A rapid growth has been noticed in various wireless technologies in recent years. This has resulted in an increase in demand for wireless data services and multimedia application such as VoIP, streaming audio and video [12]. In order to provide good service and to meet the user demands, research has been in progress both in wireless technologies and VoIP network system. VoIP is becoming more and more popular especially after the deployment of WiMAX network in many countries [13]. Different aspects of VoIP over WiMAX have been addressed by researchers. The authors in [14] have investigated the data and voice support in the WiMAX network. The aim of their work was to examine the QoS deployment over WiMAX network and compare the performance obtained using two different WiMAX services classes i.e. UGS and ertPS. The author in [15] has pointed out different factors like delay, jitter and packet losses and discussed how WiMAX network can deal with them.

\section{QoS Service Classes in WiMAX}

WiMAX accords network operators the opportunity to deliver a wealth of services to distinguish their offerings and attract a tiered range of subscribers. It features a diversity of flow types that can be used to improve performance for voice, video, and data. For example, a user having a Voice over IP (VoIP) application needs a real-time data stream unlike another one transferring an FTP file. The IEEE802.16 WiMAX standard offers four categories for the prioritization of traffic named as nsolicited Grant Service (UGS), Real-Time Polling Service (rtPS), Non-Real Time Polling Service (nrtPS), and Best Effort, there is a fifth type QoS service 
class which is added in 802.16e standard, named as: extended real-time Polling Service (ertPS). IEEE 802.16 has five QoS classes [8].

\section{VoIP Transport System}

\section{Voip Technology}

VoIP uses a combination of protocols for delivering phone data over networks. Various signaling protocols are used, SIP and H.323 can be regarded as the enabler protocols for voice over IP (VoIP) services [9]. VoIP communications require these signaling systems to setup, control, initiate a session and facilitate real-time data transfer in order to provide clear communications. SIP and H.323 works in conjunction with the Real Time Transport Protocol (RTP) and the User Datagram Protocol (UDP) to transfer the voice stream. Voice data is putted in data packets using the RTP protocol. The RTP packets, enclosed inside the UDP packets, are then transferred to the receiver.

\section{VoIP Codecs}

RTP and UDP protocols are the logical choice to carry voice when TCP protocol favors reliability over timeliness. Voice signals are digitally encoded. This means that each voice signal is converted from digital to analog and back. The analog signal is firstly sampled based on a sampling rate of $8 \mathrm{KHz}, 8$ bits per sample is the most frequently cases. Next, the output is encoded according to many factors: the compression rate and the framing time or the frames length. Finally, one or more of these frames are encapsulated into an RTP/UDP/IP packet for transmission over the network. All these practices are accomplished by one of various audio codecs, each of which vary in the sound quality, the bandwidth required, the computational requirements, encoding algorithm and coding delay $[9,10,11]$ :

- G.711 is the default standard for all vendors, very low processor requirements. This standard digitizes voice into $64 \mathrm{Kbps}$ and does not compress the voice, It performs best in local networks where we have lots of available bandwidth.

- G.729 is supported by many vendors for compressed voice operating at 8 Kbps. Excellent bandwidth utilization and Error tolerant with quality just below that of G.711.

- G.723.1 was once the recommended compression standard. It operates at 6.3 and $5.3 \mathrm{Kbps}$. High compression with high quality audio. Although this standard reduces bandwidth consumption, voice is much poorer than with G.729 and is not very popular for VoIP.

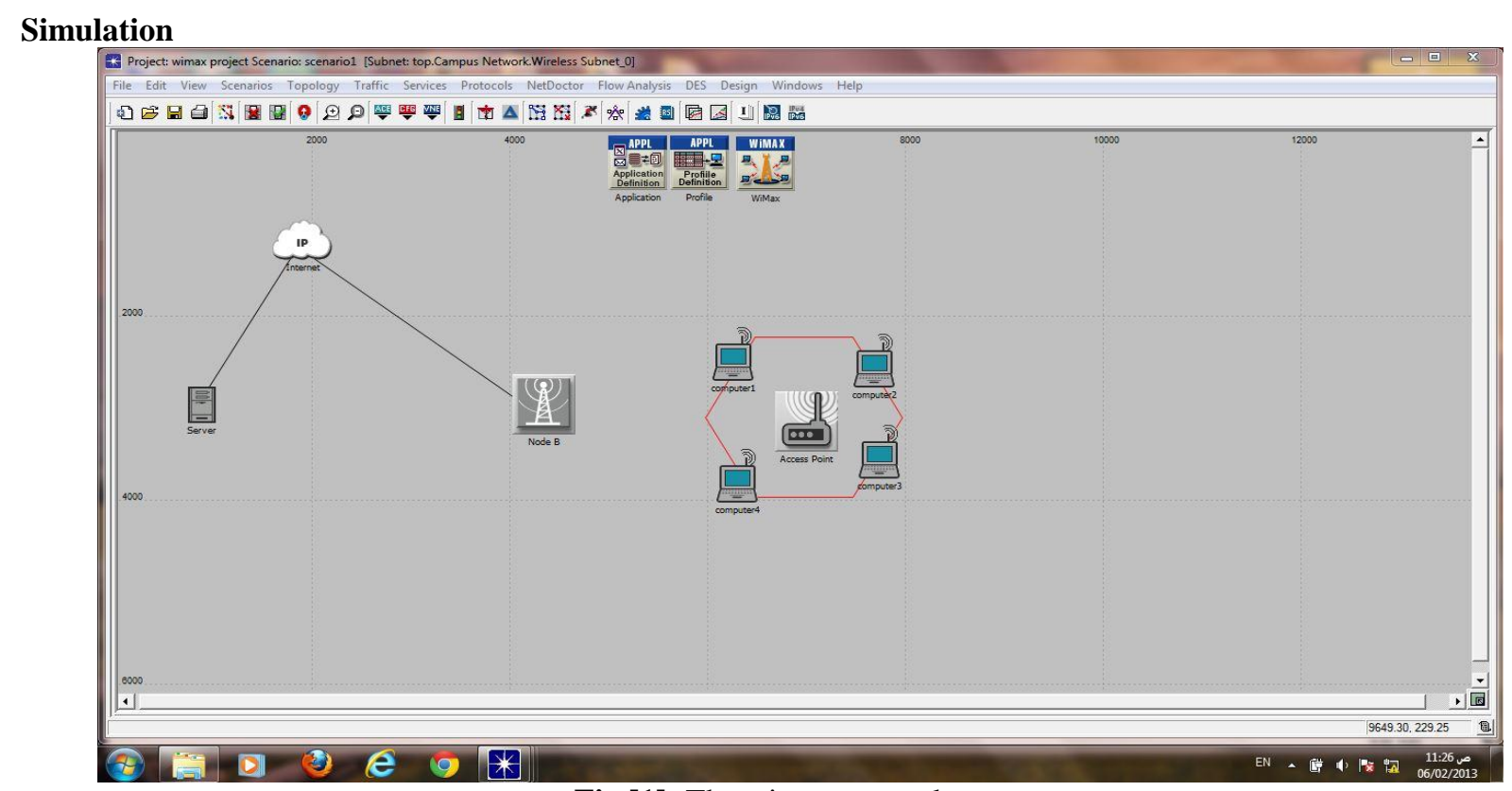

Fig [1]: The wimax network 


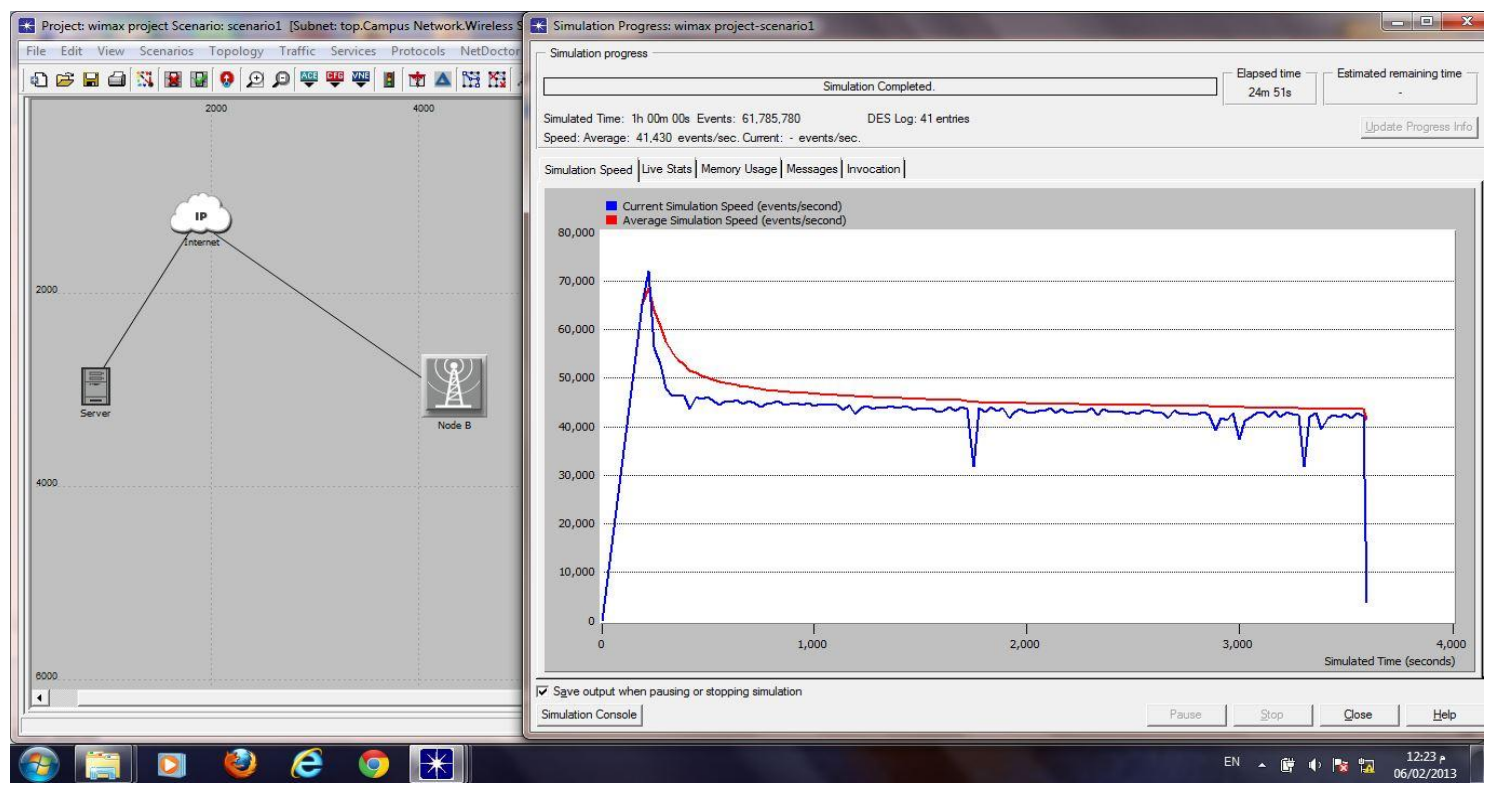

Fig 2: simulation progress

\section{Performance Metrics}

In this study, we used the following multi metrics to evaluate the performance of WiMAX in terms of end to end QoS for VoIP, according to figures below .As VoIP packets travel through a network, there evidently are some congestion and channel related losses. In addition, the packets suffer delay, depending on the congestion at the intermediate routers. Both the loss and delay of packets adversely affect the quality of VoIP calls, which is generally expressed.

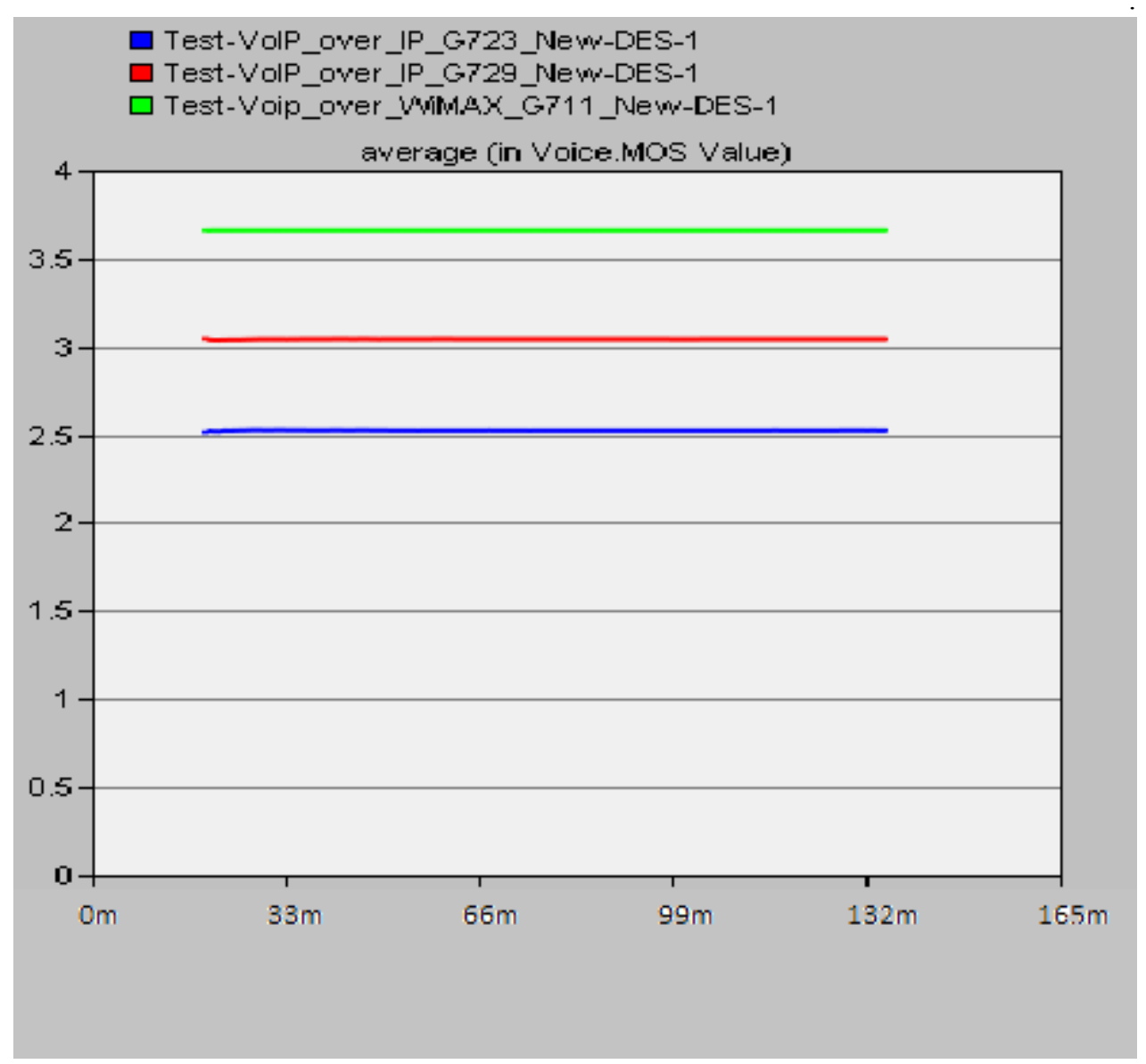

Fig 3: Mean Opinion Score MOS 


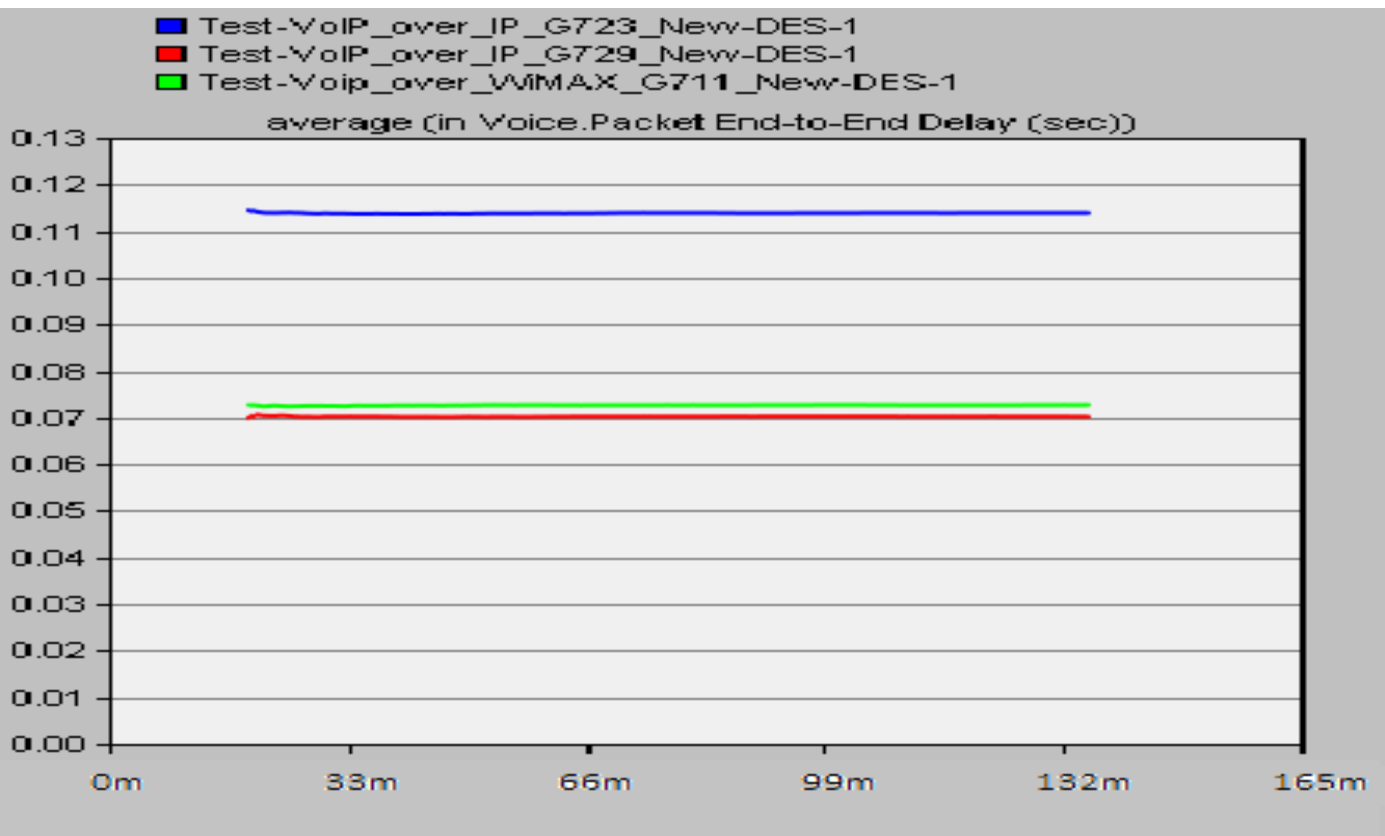

Fig. 4: Voice Packet End-to-End Delay for G.711, G.723 and G.729

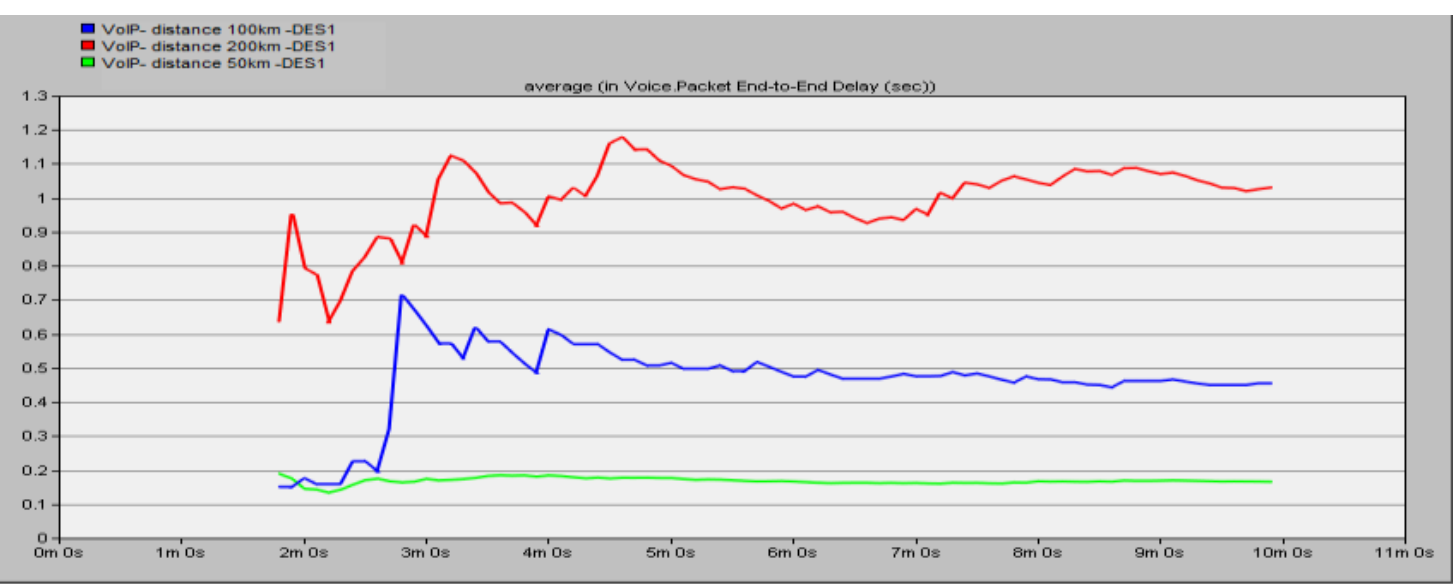

Fig 5: Average in voice packet End to End Delay

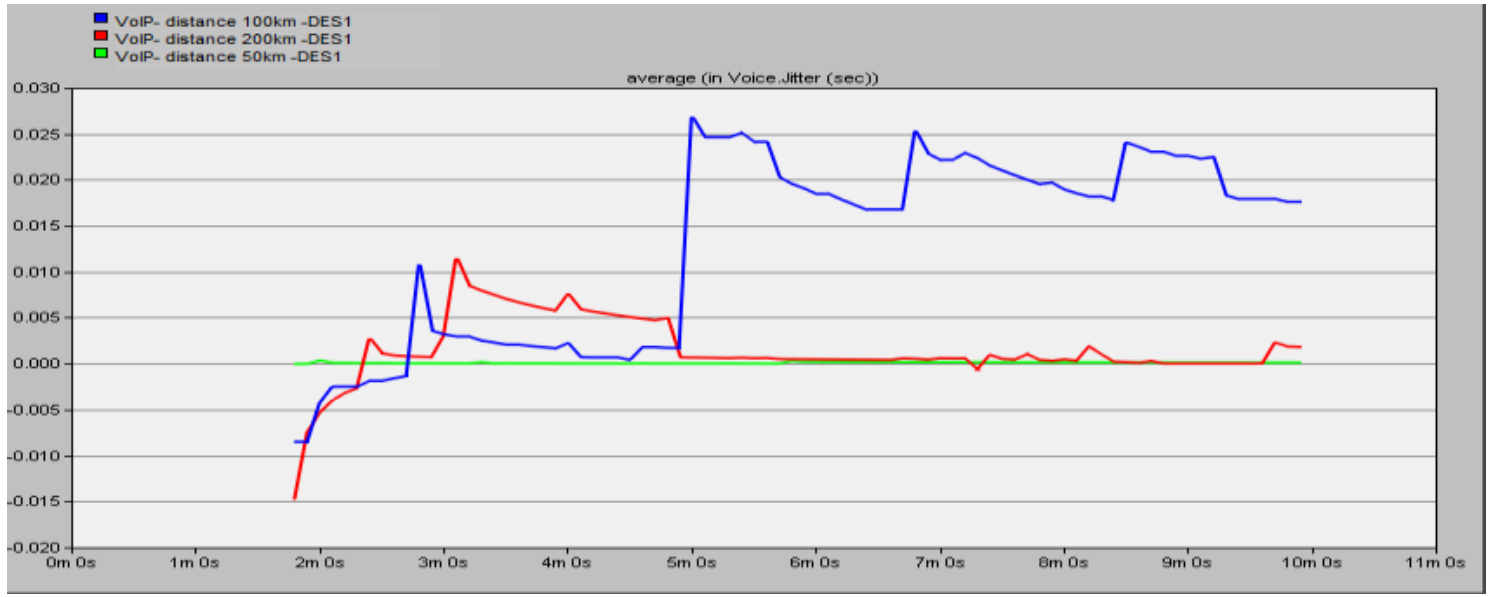

Fig 6: Average in voice jitter 


\section{Analysis:}

Figure1 shown below is the simulation set up which is used for WiMAX network with multiple subscriber station which is in the range of Node B is deployed using the wireless simulator OPNET. The Base Station and the core network are connected to each other by server backbone through an IP backbone and also through an ASN gateway which controls the mobile nodes mobility. The server backbone is also connected further to the voice server which is configured as the SIP server. Service Provider Company network is formed by the combination of the Base Station, IP backbone, ASN gateway, server backbone and the voice server. To control the mobility of the mobile nodes a Generic Routing Encapsulation tunnel is set between the Base Station and the ASN gateway Now the various voice codec's are used for voice over IP calls and the variation in the following parameters is studied i.e. Also very few voice codec's could be modelled that are G.723.1 with $5.3 \mathrm{kbps}, \mathrm{G} 726$ with $32 \mathrm{kbps}$ and $\mathrm{G} 728$ with $16 \mathrm{kbps}$

Fig 2 shown simulation progress.. Also very few voice

Fig 3: present the MOS for G.711,G.729,G.723. It is observed that, the MOS significantly increases (above 3.5), indicating the improvement of the call quality of VoIP streams and according to this the voice over IP in WIMAX for G.711codec is the best.

The effect of delay In a VoIP system, the total mouth to ear delay is composed of three components: codec delay, play out delay , and network delay Codec delay represents the algorithmic and packetization delay associated With the codec and varies from codec to codec. For example the G.729a codec introduces a delay .Playout delay is the delay associated with the receiver side buffer required to smooth out the jitter for the arriving packet streams. Network delay is the one way transit delay across the IP transport network from one gateway to another Thus, the total delay is.

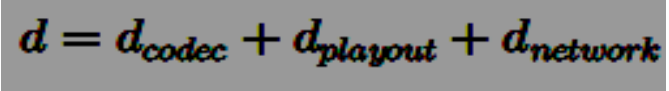

Fig 4 shown Packet End to End delay : This parameter gives the total voice packet delay i.e. the mouth to ear delay between the users. In all simulations, the mean end to end delay is shown for the all the users in the network research have confirm that packet delay of $100 \mathrm{~ms}$ doesn't do any problem, but if the delay grow up to $150 \mathrm{~ms}$ the voice signal is damage. The service providers have to guarantee that the delay happen is equal or less than 100ms .and according to fig 4 the delay in G.729 and G.723 gives best result .

Fig 5,6 shown Average in voice packet End to End Delay and average in voice jitter according three distances $50,100,200 \mathrm{~km}$ and at $50 \mathrm{~km}$ low jitter and delay.

\section{Conclusion}

This implementation was performed using OPNET. In our OPNET modeler implementation of VOIP over WiMAX, there are some parameters specified as IEEE802.16 parameters to evaluate QoS and some specified as Voice parameter's with different codecs. The simulation study was carried out to evaluate the performance of VoIP over the WiMAX networks. Different parameters such as MOS value, packet end-to-end delays, jitter and voice packets end to end delay according to three distance $(50,100,200 \mathrm{~km})$, were used to measure the performance of VoIP over WiMAX. Three voice codecs i.e G.711, G.723 and G.729 were simulated in order to find the most appropriate voice codec for VoIP over WiMAX network. The simulation results showed that VoIP performed best under the G.711 codec as compared to the G.723 and G.729 codecs. The research findings also show that VoIP applications can perform better under the G.729 codec and the distance has large effect on voice quality. According to fig 5,6 the end to end delay and jitter increasing if the distance increased

Based on the results obtained in this paper through simulation, the options presented in this thesis can be seen as positive solutions

\section{Reference}

[1]. IEEE Standard for Local and Metropolitan Area Networks Part 16: Air Interface for Fixed Broadband Wireless Access Systems, IEEE Standard 802.16-2001 Working Group Std., 2002.

[2]. E. Halepovic, M. Ghaderi and C. Williamson, "Multimedia application performance on a WiMAX network," in Proc. Of Annual Multimedia Computing and Networking Symposium,2009 [3] I. Adhicandra, "Measuring data and voip traffic in wimax networks," Arxiv Preprint arXiv:1004.4583, 2010.

[3]. A.P. Markopoulou, F.A. Tobagi, and M.J. Karam, "Assessment of VoIP Quality over Internet Backbones," Proc. IEEE INFOCOM '02, vol. 1, pp. 150-159, 2002.

[4]. B. L. Dang, Y. Zhao, V. Prasad, I. Niemegeers, "Performance study of scheduling algorithms for WiMAX networks", OPNETWORK 2007.

[5]. C. Boutremans, G. Iannaccone, and C. Diot, "Impact of Link Failures on VoIP Performance," Proc. 12th Int'l Workshop Network and Operating Systems Support for Digital Audio and Video, pp. 63-71, 2002. 
[6]. D.T. Chen, N. Natarajan, and Y. Sun, "On the Simulation, Modeling, and Performance Analysis of an 802.16E Mobile Broadband Wireless Access System," Comm. and Computer Networks, 2005.

[7]. Z. Abichar, Y. Peng and J. Morris Chang, "WiMax: The Emergence of Wireless Broadband", IT Professional, Vol. 8, Issue. 4, pp. 44-48, Doi:10.1109/MITP.2006.99, July-Aug. 2006.

[8]. S. Karapantazis and F.-N. Pavlidou, "VoIP: A comprehensive survey on a promising technology", Computer Networks, Vol. 53, Issue 12, pp. 2050-2090, 2009.

[9]. A. Kumar and S. Ganesh Thorenoor, "Analysis of IP Network for different Quality of Service", International Symposium on Computing, Communication, and Control (ISCCC 2009) Proc. of CSIT Vol.1 (2011) () (2011) IACSIT Press, Singapore.

[10]. T. Zourzouvillys and E. Rescorla, "An introduction to standards-based VoIP: SIP, RTP, and friends", Internet Computing, IEEE, Vol. 14, Issue 2, pp. $69-73,2010$.

[11]. S. Sengupta, M. Chatterjee and S. Ganguly, "Improving quality of VoIP streams over WiMax," Computers, IEEE Transactions on, vol. 57, pp. 145-156, 2008

[12]. E. Halepovic, M. Ghaderi and C. Williamson, "Multimedia application performance on a WiMAX network," in Proc. Of Annual Multimedia Computing and Networking Symposium, 2009, .

[13]. I. Adhicandra, "Measuring data and voip traffic in wimax networks," Arxiv Preprint arXiv:1004.4583, 2010.

[14]. Tucker, E. 2006. Can voice be the killer App for WiMAX. Available from: http://www.openbasestation.org/Newsletters/November2006/A perto.htm [Last Accessed on 15th March, 2012]

[15]. ITU-T Recommendation G.107, The E-Model: A Computational Model for Use in Transmission Planning, Dec. 1998. 